Portland State University

PDXScholar

Electrical and Computer Engineering Faculty

Publications and Presentations

7-1-1981

\title{
Synthesis of Gaussian Beam Optical Systems
}

Lee W. Casperson

Portland State University

Follow this and additional works at: https://pdxscholar.library.pdx.edu/ece_fac

Part of the Electrical and Computer Engineering Commons

Let us know how access to this document benefits you.

\section{Citation Details}

Lee W. Casperson. Synthesis of Gaussian beam optical systems. Applied Optics, Volume 20, Number 13 (July 1981), pp. 2243-2249.

This Article is brought to you for free and open access. It has been accepted for inclusion in Electrical and Computer Engineering Faculty Publications and Presentations by an authorized administrator of PDXScholar. Please contact us if we can make this document more accessible: pdxscholar@pdx.edu. 


\title{
Synthesis of Gaussian beam optical systems
}

\author{
Lee W. Casperson
}

\begin{abstract}
Systematic procedures are presented for determining the the optical components needed to obtain an arbi-
\end{abstract} trary transformation of a propagating light ray or Gaussian beam.

\section{Introduction}

A fundamental problem of optics and quantum electronics concerns the propagation of optical signals from one reference plane to another. A variety of techniques have been developed for addressing this problem, and all these techniques are based directly or remotely on Maxwell's equations. Of particular interest in the present study are the propagation methods that are based on a type of $2 \times 2$ transfer matrix. ${ }^{1}$ The basic idea is that the overall transfer matrix for an optical system can be represented as the ordered matrix product of the transfer matrices of the individual elements that constitute the system. When suitably represented, many aspects of a ray or beam can be propagated through an optical system by means of simple operations on the corresponding matrix.

Previous studies of matrix methods in optics have been directed primarily at the analysis of existing or postulated optical systems. In a typical problem one might be given a sequence of lenses, mirrors, lenslike media, etc. and be required to find a relationship between an input Gaussian beam and the corresponding output beam. Based on experience with many such systems one is sometimes able to guess the type of system that is needed to obtain a required beam transformation. The emphasis in the present study is on synthesis. It ought not to be necessary to rely on experience or good luck to design an optical system that will produce some required transformation of a ray or beam. Systematic procedures are developed for finding the simplest possible system that will yield a specified

The author is with University of California, School of Engineering \& Applied Science, Los Angeles, California 90024.

Received 10 February 1981.

0003-6935/81/132243-07\$00.50/0.

(C) 1981 Optical Society of America. transformation. One finds, for example, that for some transformations only one lens may be needed, while for others a minimum of two or three may be required.

In Sec. II are mentioned some ways in which matrices can be applied to optical problems. From these formulas one can deduce the matrix needed for a desired transformation. The following sections address the question of whether such a matrix can be synthesized using real optical components. In Sec. III, it is shown that almost any $2 \times 2$ matrix that can be encountered in optics is factorable into at most four primitive matrices of three basic types. It is demonstrated in Sec. IV how each of these primitive matrices can be realized using actual laboratory components. A basic result is that any complex $A B C D$ matrix can be synthesized for beam optical applications provided that the determinant is real and positive. These techniques are illustrated in Sec. V by investigating some practical systems in which the transfer matrix is equal to the identity matrix. Such systems would be invisible in terms of measurements made at the input and output reference planes.

\section{Propagation of Rays and Beams}

An exact description of the electromagnetic field distribution in a resonator or other optical system requires a solution of Maxwell's equations subject to any relevant boundary conditions. For practical applications a variety of approximate solution techniques are available for obtaining useful information about such field distributions. Many aspects of optical systems can be derived simply from geometrical optics without considering diffraction or other physical optics effects. For paraxial light rays in geometrical optics, the ray propagation formulas can be reduced to an equation having the well-known form ${ }^{1}$

$$
\left(\begin{array}{l}
r_{2} \\
r_{2}^{\prime}
\end{array}\right)=\left(\begin{array}{ll}
A & B \\
C & D
\end{array}\right)\left(\begin{array}{l}
r_{1} \\
r_{1}^{\prime}
\end{array}\right),
$$

where the $r$ and $r^{\prime}$ coordinates represent, respectively, the radial position and slope of a light ray at reference plane 1 or 2 . The $A B C D$ matrix may represent a single 
optical component, or it may refer to the product matrix corresponding to a sequence of optical elements. In a typical problem of optical analysis one is given a sequence of optical elements and is required to find a relationship between the input and output light rays. The solution then reduces to a matter of matrix multiplication. Many features of laser resonators can also be deduced from such ray optical techniques.

The problem of synthesis is of exactly the opposite nature. We assume that there is some desired relationship between the positions and slopes of the input and output light rays, and the necessary optical system is to be determined. For example, it might be desired to design an optical system that would double the displacement and halve the slope of a light ray in a distance of $1 \mathrm{~m}$. Clearly this problem would involve finding a set of factors of the matrix

$$
\left(\begin{array}{ll}
A & B \\
C & D
\end{array}\right)=\left(\begin{array}{cc}
2 & 0 \\
0 & 0.5
\end{array}\right)
$$

where each factor corresponds to some realizable optical element, and the total length constraint is also somehow satisfied. The possible solutions to problems of this type are discussed in this paper.

Another class of problems involves the propagation of Gaussian beams through paraxial optical systems. The basic parameters of a Gaussian beam are the 1/e amplitude spot size $w$ and the phase front curvature $R$, and these quantities may be combined to form a complex beam parameter $Q$ or $q$, given by

$$
\frac{Q}{k_{0}}=\frac{1}{q}=\frac{1}{R}-\frac{i \lambda}{\pi w^{2}}
$$

where $\lambda$ is the wavelength and $k_{0}$ is the propagation constant in the material of interest.

The propagation formula for such a beam is the Kogelnik transformation ${ }^{2}$

$$
\frac{1}{q_{2}}=\frac{C+D / q_{1}}{A+B / q_{1}}
$$

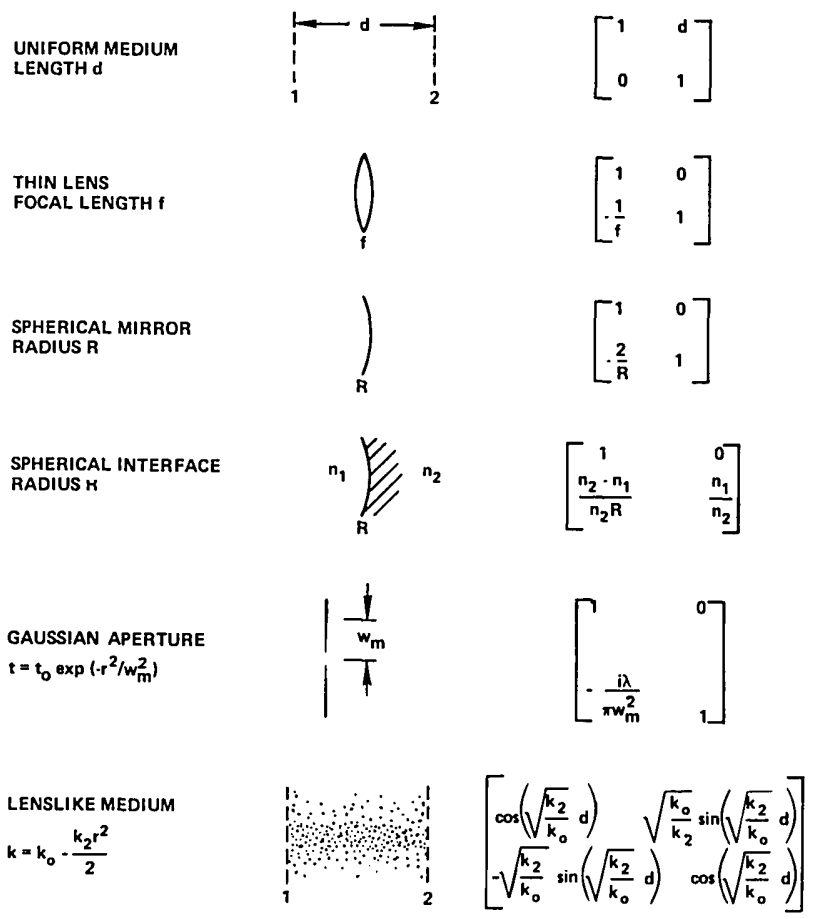

Fig. 1. Matrices for a Gaussian beam incident from the left.

where $A, B, C$, and $D$ are the elements of a beam matrix describing the propagation from reference plane 1 to reference plane 2. For our piresent synthesis interests, it is assumed that the input beam $q_{1}$ is known, as is the output beam $q_{2}$. Then the initial stages of the synthesis problem involved finding the appropriate matrix elements for use in Eq. (4), and some of the possible elements are listed for reference in Fig. 1. Most of these matrices were discussed by Kogelnik, ${ }^{1}$ but the complex lenslike medium ${ }^{3,4}$ and the Gaussian transmission filter ${ }^{5}$ are later additions.

For general complex values of the matrix elements, Eq. (3) can be written in the expanded form

$$
\frac{1}{R_{2}}-\frac{i \lambda}{\pi w_{2}^{2}}=\frac{\left(C_{r}+\frac{D_{r}}{R_{1}}+\frac{D_{i} \lambda}{\pi w_{1}^{2}}\right)+i\left(C_{i}+\frac{D_{i}}{R_{1}}-\frac{D_{r} \lambda}{\pi w_{1}^{2}}\right)}{\left(A_{r}+\frac{B_{r}}{R_{1}}+\frac{B_{i} \lambda}{\pi w_{1}^{2}}\right)+i\left(A_{i}+\frac{B_{i}}{R_{1}}-\frac{B_{r} \lambda}{\pi w_{1}^{2}}\right)} .
$$

If this result is separated into its real and imaginary parts, one obtains

$$
\begin{aligned}
& \frac{1}{R_{2}}=\frac{\left(C_{r}+\frac{D_{r}}{R_{1}}+\frac{D_{i} \lambda}{\pi w_{1}^{2}}\right)\left(A_{r}+\frac{B_{r}}{R_{1}}+\frac{B_{i} \lambda}{\pi w_{1}^{2}}\right)+\left(C_{i}+\frac{D_{i}}{R_{1}}-\frac{D_{r} \lambda}{\pi w_{1}^{2}}\right)\left(A_{i}+\frac{B_{i}}{R_{1}}-\frac{B_{r} \lambda}{\pi w_{1}^{2}}\right)}{\left(A_{r}+\frac{B_{r}}{R_{1}}+\frac{B_{i} \lambda}{\pi w_{1}^{2}}\right)^{2}+\left(A_{i}+\frac{B_{i}}{R_{1}}-\frac{B_{r} \lambda}{\pi w_{1}^{2}}\right)^{2}}, \\
& \frac{\lambda}{\pi w_{2}^{2}}=\frac{-\left(C_{i}+\frac{D_{i}}{R_{1}}-\frac{D_{r} \lambda}{\pi w_{1}^{2}}\right)\left(A_{r}+\frac{B_{r}}{R_{1}}+\frac{B_{i} \lambda}{\pi w_{1}^{2}}\right)+\left(C_{r}+\frac{D_{r}}{R_{1}}+\frac{D_{i} \lambda}{\pi w_{1}^{2}}\right)\left(A_{i}+\frac{B_{i}}{R_{1}}-\frac{B_{r} \lambda}{\pi w_{1}^{2}}\right)}{\left(A_{r}+\frac{B_{r}}{R_{1}}+\frac{B_{i} \lambda}{\pi w_{1}^{2}}\right)^{2}+\left(A_{i}+\frac{B_{i}}{R_{1}}-\frac{B_{r} \lambda}{\pi w_{1}^{2}}\right)^{2}} .
\end{aligned}
$$


These are two equations to be solved for the real and imaginary parts of the matrix elements, i.e., two equations in eight unknowns. Several additional constraints may follow from other considerations.

Besides the phase front curvature and spot size, which are characterized by the beam parameter $q$, there are other properties of Gaussian beams that may also be of interest. In many cases, these properties can be used to derive additional constraints on the elements of the beam matrix. For example, one of several useful sets of higher-order beam modes involves the astigmatic off-axis Hermite-Gaussian functions of real argument $^{6}$ :

$$
\begin{aligned}
E(x, y, z)= & E_{0} H_{m}\left(2^{1 / 2} \frac{x}{w_{x}}\right) H_{n}\left(2^{1 / 2} \frac{y}{w_{y}}\right) \\
& \times \exp \left\{-i\left[k_{0} z+\frac{Q_{x}(z) x^{2}}{2}+\frac{Q_{y}(z) y^{2}}{2}\right.\right. \\
& \left.\left.+S_{x}(z) x+S_{y}(z) y+P(z)\right]\right\} .
\end{aligned}
$$

The subscripts $x$ and $y$ denote the fact that the $x$ and $y$ variations may be unequal in an astigmatic system. The $S$ parameters show the displacement of the beam away from the $z$ axis. The $x$ displacement of the amplitude center is given by $d_{x a}=-S_{x i} / Q_{x i}$, and the $x$ displacement of the phase center is given by $d_{x p}=$ $-S_{x r} / Q_{x r}$. The subscripts $i$ and $r$ indicate, respectively, the imaginary and real parts of the parameters $S_{x}$ and $Q_{x}$, and similar relations apply to the functions $S_{y}$ and $Q_{y}$. The parameter $P$ measures the relative on-axis complex phase of the propagating beam. This is the mode dependent complex phase shift, excluding the plane wave phase $-i k_{0} z$, reflection losses at dielectric boundaries, unknown constant phase shifts at thin lenses, etc.

The propagation of the beam displacement and phase through an optical system can also be expressed as simple transformations involving the elements of the corresponding beam matrix. Thus the complex parameter $S_{x}$ propagates according to ${ }^{7}$

$$
S_{x_{2}}=\frac{S_{x_{1}}}{A_{x}+B_{x} / q_{x_{1}}}
$$

with a similar formula for $S_{y}$. These results would be useful in beam scanning systems. If the deflection properties of a system are specified, the real and imaginary parts of Eq. (9) can be used to obtain additional constraints on the matrix elements. Similarly, the propagation of the phase parameter is found to obey the relation

$$
\begin{aligned}
P_{2}-P_{1}= & -\frac{i}{2} \operatorname{Re} \ln \left(A_{x}+\frac{B_{x}}{q_{x_{1}}}\right)+\left(m+\frac{1}{2}\right) \operatorname{Im} \ln \left(A_{x}+\frac{B_{x}}{q_{x_{1}}}\right) \\
& -\frac{i}{2} \operatorname{Re} \ln \left(A_{y}+\frac{B_{y}}{q_{y_{1}}}\right)+\left(n+\frac{1}{2}\right) \operatorname{Im} \ln \left(A_{y}+\frac{B_{y}}{q_{y_{1}}}\right) \\
& -\frac{S_{x 1}^{2}}{2 k_{0_{1}}} \frac{B_{x}}{A_{x}+B_{x} / q_{x_{1}}}-\frac{S_{y_{1}}^{2}}{2 k_{0_{1}}} \frac{B_{y}}{A_{y}+B_{y} / q_{y_{1}}},
\end{aligned}
$$

where $k_{0_{1}}$ is the propagation constant at the input of the optical element or system $\left(k_{0}\right.$ changes when crossing a dielectric boundary). Phase formulas of this type would be useful in interferometry and resonator mode frequency studies. For our synthesis interests it is clear that any phase shift constraints can also be translated into conditions on the matrix elements by means of Eq. (10).

As mentioned above the most general complex beam matrix encountered in a synthesis problem would involve eight unknowns. The conditions on these unknowns that have been discussed so far all follow from the propagation formulas for Gaussian beams. Other types of conditions can also arise. For example, it will be seen that the determinant of the beam matrix for an optical system is always equal to the ratio of the index of refraction at the input plane to the index of refraction at the output. Therefore, any physically realizable system must satisfy the condition

$$
A D-B C=n_{1} / n_{2} .
$$

The real and imaginary parts of this equation provide two more constraints. In a resonator or waveguide synthesis problem one might require that the resulting modes be stable with respect to perturbations. In this case the matrix elements would have to satisfy the condition ${ }^{7}$

$$
1 \leqslant\left|\frac{A+D}{2} \pm\left[\left(\frac{A+D}{2}\right)^{2}-1\right]^{1 / 2}\right|
$$

and other conditions could be deduced for high loss resonators. ${ }^{8}$

Most often one would also be inclined to require that the matrix elements be strictly real. Complex lenslike media are typically more costly and complicated to fabricate, and Gaussian transmission filters always reduce the total power in the transmitted beam. If the matrix elements are required to be real, four of our eight unknowns are abruptly set equal to zero. Still other constraints might result from other practical considerations. For example, it might be specified that the optical system has a length of $l$ between the reference planes or that the spot size and beam displacement are everywhere less than some specified value. For the moment, it is simply assumed that the matrix elements of the desired transformation have, by one means or another, been determined.

\section{Factoring the Matrix}

Once the matrix elements are known, one is left with the task of finding actual optical components from Fig. 1 , which when placed in sequence yield the required matrix. As a starting point, it may be observed that, with the possible exception of the lenslike medium, all the fundamental elements from Fig. 1 can be readily expressed as products of the following three forms:

$$
\begin{aligned}
& \alpha=\left(\begin{array}{ll}
1 & \alpha \\
0 & 1
\end{array}\right) ; \\
& \beta=\left(\begin{array}{ll}
1 & 0 \\
\beta & 1
\end{array}\right) ; \\
& \gamma=\left(\begin{array}{ll}
1 & 0 \\
0 & \gamma
\end{array}\right) .
\end{aligned}
$$

It is thus reasonable to inquire how broad a class of 
$A B C D$ matrices can be represented as a product of these matrices. In this connection one obtains the following theorem:

THEOREM: Any nondegenerate $2 \times 2$ matrix can be represented as a product of at most four matrices of $\alpha, \beta$, and $\gamma$ type.

By degenerate matrices would here be meant certain special cases in which simpler factorizations are possible or more complicated factorizations are required. All such cases are discussed in this section. The preceding theorem is proved if one can find any example of four matrices, which when multiplied together produce an arbitary $A B C D$ matrix. If the $C$ element is unequal to zero, one possibility is the following:

$$
\begin{aligned}
& \left(\begin{array}{ll}
A & B \\
C & D
\end{array}\right)=\left(\begin{array}{ll}
1 & \alpha \\
0 & 1
\end{array}\right)\left(\begin{array}{ll}
1 & 0 \\
\beta & 1
\end{array}\right)\left(\begin{array}{ll}
1 & 0 \\
0 & \gamma
\end{array}\right)\left(\begin{array}{ll}
1 & \alpha^{\prime} \\
0 & 1
\end{array}\right) \\
= & \left(\begin{array}{cc}
1 & (A-1) / C \\
0 & 1
\end{array}\right)\left(\begin{array}{ll}
1 & 0 \\
C & 1
\end{array}\right)\left(\begin{array}{cc}
1 & 0 \\
0 & A D-B C
\end{array}\right)\left(\begin{array}{cc}
1 & B+(1-A) D / C \\
0 & 1
\end{array}\right) .
\end{aligned}
$$

The validity of the factorization can be comfirmed by direct multiplication, and it is clear that each of these factors is of the $\alpha, \beta$, or $\gamma$ type.

It is also of interest to consider the uniqueness of factorizations like that given in Eq. (16). For this purpose certain preliminary considerations are required. First, it may be observed that the $\gamma$ matrix quasi-commutes with matrices of the other types. By this we mean that a product $\gamma \alpha$ (or $\gamma \beta$ ) can be replaced by a product $\alpha^{\prime} \gamma$ (or $\beta^{\prime} \gamma$ ). More specifically, one finds the following relations:

$$
\begin{aligned}
& \left(\begin{array}{ll}
1 & 0 \\
0 & \gamma
\end{array}\right)\left(\begin{array}{ll}
1 & \alpha \\
0 & 1
\end{array}\right)=\left(\begin{array}{ll}
1 & \alpha \\
0 & \gamma
\end{array}\right)=\left(\begin{array}{ll}
1 & \alpha / \gamma \\
0 & 1
\end{array}\right)\left(\begin{array}{ll}
1 & 0 \\
0 & \gamma
\end{array}\right), \\
& \left(\begin{array}{ll}
1 & 0 \\
0 & \gamma
\end{array}\right)\left(\begin{array}{ll}
1 & 0 \\
\beta & 1
\end{array}\right)=\left(\begin{array}{ll}
1 & 0 \\
\beta \gamma & \gamma
\end{array}\right)=\left(\begin{array}{ll}
1 & 0 \\
\beta \gamma & 1
\end{array}\right)\left(\begin{array}{ll}
1 & 0 \\
0 & \gamma
\end{array}\right)
\end{aligned}
$$

On the other hand, one can show that there is no corresponding commutation relation between matrices of types $\alpha$ and $\beta$. In the nontrivial situations where the elements $\alpha$ and $\beta$ are unequal to zero, such a commutation relation would take the form

$$
\left(\begin{array}{ll}
1 & \alpha \\
0 & 1
\end{array}\right)\left(\begin{array}{ll}
1 & 0 \\
\beta & 1
\end{array}\right)=\left(\begin{array}{ll}
1 & 0 \\
\beta^{\prime} & 1
\end{array}\right)\left(\begin{array}{ll}
1 & \alpha^{\prime} \\
0 & 1
\end{array}\right)
$$

Carrying out the multiplication leads to

$$
\left(\begin{array}{cc}
1+\alpha \beta & \alpha \\
\beta & 1
\end{array}\right)=\left(\begin{array}{cc}
1 & \alpha^{\prime} \\
\beta^{\prime} & 1+\alpha^{\prime} \beta^{\prime}
\end{array}\right)
$$

from which follow the equations $\alpha=\alpha^{\prime}, \beta=\beta^{\prime}$, and $\alpha \beta$ $=\alpha^{\prime} \beta^{\prime}=0$. But this is a contradiction since by assumption $\alpha$ and $\beta$ are nonzero. Hence no such commutation is possible.

Using the previous commutation considerations, it is now straightforward to investigate the uniqueness of expansions like that given in Eq. (16). The proof is by contradiction, and we start by postulating that two different factorizations of the same matrix exist in the forms

$$
\alpha_{1} \beta_{1} \gamma_{1} \alpha_{1}^{\prime}=\alpha_{2} \beta_{2} \gamma_{2} \alpha_{2}^{\prime}
$$

These forms are exactly equivalent to Eq. (16) and are similar to several other possible factorizations. First, it may be noted that only the matrices of $\gamma$ type have determinants different from unity. Since the determinant of a matrix product is equal to the product of the determinants, it follows that $\gamma_{1}=\gamma_{2}=\gamma$.

It can also be observed that all the component matrices in Eq. (21) possess inverses and that matrix multiplication is associative $[\alpha(\beta \gamma)=(\alpha \beta) \gamma]$. If this equation is premultiplied by $\alpha_{1}^{-1}$ and postmultiplied by $\left(\gamma \alpha_{2}^{\prime}\right)^{-1}$, the result is

$$
\beta_{1} \gamma \alpha_{1}^{\prime}\left(\gamma \alpha_{2}^{\prime}\right)^{-1}=\alpha_{1}^{-1} \alpha_{2} \beta_{2}
$$

But the inverse of an $\alpha$ matrix is still an $\alpha$ matrix, and the product of two $\alpha$ matrices is also an $\alpha$ matrix. Therefore, we can define two new $\alpha$ matrices $\alpha_{3}=$ $\gamma \alpha_{1}^{\prime}\left(\gamma \alpha_{2}^{\prime}\right)^{-1}$ and $\alpha_{4}=\alpha_{1}^{-1} \alpha_{2}$, and Eq. (22) reduces to

$$
\beta_{1} \alpha_{3}=\alpha_{4} \beta_{2}
$$

But from the noncommutativity of $\alpha$ and $\beta$ matrices discussed previously, it follows that both $\alpha_{3}$ and $\alpha_{4}$ are equal to the identity matrix and, therefore, that $\beta_{1}=\beta_{2}$. Also, $\alpha_{4}$ can only equal the identity matrix if $\alpha_{1}=\alpha_{2}$, and $\alpha_{3}$ can only equal the identify matrix if $\gamma \alpha_{1}^{\prime}=\gamma \alpha_{2}^{\prime}$ or $\alpha_{1}^{\prime}=\alpha_{2}^{\prime}$. Therefore, contrary to the initial assumption of Eq. (21), the expansion of an arbitary matrix in the $\alpha \beta \gamma \alpha^{\prime}$ form is unique. It remains to be seen whether expansions in other product forms might still be possible.

Because of the quasi-commutative property of the $\gamma$ matrix it follows immediately that there are three other variations of the factorization given in Eq. (16), depending on the position in the product of the matrix $\gamma$. For completeness we write down all four possibilities:

$$
\begin{aligned}
\left(\begin{array}{ll}
A & B \\
C & D
\end{array}\right) & =\left(\begin{array}{cc}
1 & 0 \\
0 & A D-B C
\end{array}\right)\left(\begin{array}{cc}
1 & (A-1)(A D-B C) / C \\
0 & 1
\end{array}\right)\left(\begin{array}{cc}
1 & 0 \\
C /(A D-B C) & 1
\end{array}\right)\left(\begin{array}{cc}
1 B+(1-A) D / C \\
0
\end{array}\right) \\
& =\left(\begin{array}{cc}
1 & (A-1) / C \\
0 & 1
\end{array}\right)\left(\begin{array}{cc}
1 & 0 \\
0 & A D-B C
\end{array}\right)\left(\begin{array}{cc}
1 & 0 \\
C /(A D-B C) & 1
\end{array}\right)\left(\begin{array}{cc}
1 & B+(1-A) D / C \\
0 & 1
\end{array}\right) \\
& =\left(\begin{array}{cc}
1 & (A-1) / C \\
0 & 1
\end{array}\right)\left(\begin{array}{ll}
1 & 0 \\
C & 1
\end{array}\right)\left(\begin{array}{cc}
1 & 0 \\
0 & A D-B C
\end{array}\right)\left(\begin{array}{cc}
1 & B+(1-A) D / C \\
0 & 1
\end{array}\right) \\
& =\left(\begin{array}{cc}
1 & (A-1) / C \\
0 & 1
\end{array}\right)\left(\begin{array}{ll}
1 & 0 \\
C & 1
\end{array}\right)\left(\begin{array}{cc}
1 & {[B+(1-A) D / C] /(A D-B C)} \\
0 & 1
\end{array}\right)\left(\begin{array}{cc}
1 & 0 \\
0 & A D-B C
\end{array}\right) .
\end{aligned}
$$


Except for the position of the $\gamma$ matrix these four expansions all have the same basic $\alpha \beta \alpha^{\prime}$ form.

In a similar manner, one finds that there are four additional expansions having a basic $\beta \alpha \beta^{\prime}$ form. These are

$$
\begin{aligned}
\left(\begin{array}{ll}
A & B \\
C & D
\end{array}\right) & =\left(\begin{array}{cc}
1 & 0 \\
0 & A D-B C
\end{array}\right)\left(\begin{array}{cc}
1 & 0 \\
{[C+(1-A) D / B] /(A D-B C)} & 1
\end{array}\right)\left(\begin{array}{ll}
1 & B \\
0 & 1
\end{array}\right)\left(\begin{array}{cc}
1 & 0 \\
(A-1) / B & 1
\end{array}\right) \\
& =\left(\begin{array}{cc}
1 & 0 \\
C+(1-A) D / B & 1
\end{array}\right)\left(\begin{array}{cc}
1 & 0 \\
0 & A D-B C
\end{array}\right)\left(\begin{array}{cc}
1 & B \\
0 & 1
\end{array}\right)\left(\begin{array}{cc}
1 & 0 \\
(A-1) / B & 1
\end{array}\right) \\
& =\left(\begin{array}{cc}
1 & 0 \\
C+(1-A) D / B & 1
\end{array}\right)\left(\begin{array}{cc}
1 & B /(A D-B C) \\
0 & 1
\end{array}\right)\left(\begin{array}{cc}
1 & 0 \\
0 & A D-B C
\end{array}\right)\left(\begin{array}{cc}
1 & 0 \\
(A-1) / B & 1
\end{array}\right) \\
& =\left(\begin{array}{ccc}
1 & B /(A D-B C) \\
C+(1-A) D / B & 1
\end{array}\right)\left(\begin{array}{cc}
1 & 0 \\
0 & 1
\end{array}\right)\left(\begin{array}{cc}
1 & 0 \\
0 & A D-B C
\end{array}\right)
\end{aligned}
$$

Only these eight four-matrix factorizations exist for an arbitrary nondegenerate $A B C D$ matrix. Since each component matrice can in turn be factored, there are, of course, an infinite number of factorizations involving five or more component matrices.

As indicated above, there are certain special cases in which the number or form of the factorization possibilities simplifies substantially. For example, it has been tacitly assumed in the preceeding analysis that none of the initial matrix elements are equal to zero. This restriction was necessary to avoid division by zero in some of the resulting factorizations. Thus, if the $B$ element is equal to zero, the factorizations given in Eqs. (28)-(31) must be excluded, and if the $C$ element is equal to zero Eqs. (24)-(27) are not acceptable. Similarly, if the determinant vanishes, only Eqs. (26) and (29) can be considered. In the worst case, both the $B$ and $C$ elements are equal to zero [as in Eq. (2)], and no four matrix factorization is possible. We will show, however, that for such diagonal matrices a five-matrix factorization can always be found.

The first step in the decomposition of a diagonal matrix is to factor out an arbitrary matrix of $\alpha$ or $\beta$ type. The four possibilities are

$$
\begin{aligned}
\left(\begin{array}{ll}
A & 0 \\
0 & D
\end{array}\right) & =\left(\begin{array}{ll}
1 & \alpha \\
0 & 1
\end{array}\right)\left(\begin{array}{cc}
A & -\alpha D \\
0 & D
\end{array}\right) \\
& =\left(\begin{array}{cc}
A & -\alpha A \\
0 & D
\end{array}\right)\left(\begin{array}{cc}
1 & \alpha \\
0 & 1
\end{array}\right) \\
& =\left(\begin{array}{cc}
1 & 0 \\
\beta & 1
\end{array}\right)\left(\begin{array}{cc}
A & 0 \\
-\beta A & D
\end{array}\right) \\
& =\left(\begin{array}{cc}
A & 0 \\
-\beta D & D
\end{array}\right)\left(\begin{array}{ll}
1 & 0 \\
\beta & 1
\end{array}\right) .
\end{aligned}
$$

The remaining matrix can be readily factored using the matrix expansions found in Eqs. (24)-(31). For example, if one wanted a diagonal optical system to begin with a thin lens that happened to be lying around the laboratory, the appropriate starting point would be Eq. (35). The remaining factorization could use any of Eqs. (24)-(27).

An extremely important special case occurs when the $A B C D$ matrix to be factored is unimodular $(A D-B C$ $=1$ ). The importance of this case is a consequence of the following theorem:
THEOREM: The matrix corresponding to a physically realizable optical system is unimodular if and only if the output reference plane occurs in a medium having the same refractive properties as the medium surrounding the input reference plane.

This result follows from the facts that (1) the only primary nonunimodular matrix is the $\gamma$ type corresponding to a dielectric boundary, and (2) the determinant of a matrix product is equal to the product of the determinants of the component matrices. Therefore, for a sequence of $j$ dielectrics, this product is

$$
\operatorname{det}\left(\begin{array}{ll}
A & B \\
C & D
\end{array}\right)=\prod_{j} \gamma_{j}=\frac{n_{1}}{n_{2}} \cdot \frac{n_{2}}{n_{3}} \cdot \frac{n_{3}}{n_{4}} \ldots \frac{n_{j-1}}{n_{j}},
$$

from which the theorem follows.

The importance of unimodular matrices is that they correspond to most of the optical configurations that one encounters in practice. Typically, both the input and output reference planes are in air, and the determinant of the transfer matrix is unity. In addition to this practical significance, there are also substantial analytical simplications that occur when the matrix is unimodular. In particular one finds that Eqs. (24)-(27) reduce to the single equation

$$
\left(\begin{array}{ll}
A & B \\
C & D
\end{array}\right)=\left(\begin{array}{cc}
1 & (A-1) / C \\
0 & 1
\end{array}\right)\left(\begin{array}{cc}
1 & 0 \\
C & 1
\end{array}\right)\left(\begin{array}{cc}
1 & (D-1) / C \\
0 & 1
\end{array}\right)
$$

and Eqs. (28)-(31) reduce to

$$
\left(\begin{array}{ll}
A & B \\
C & D
\end{array}\right)=\left(\begin{array}{cc}
1 & 0 \\
(D-1) / B & 1
\end{array}\right)\left(\begin{array}{ll}
1 & B \\
0 & 1
\end{array}\right)\left(\begin{array}{cc}
1 & 0 \\
(A-1) / B & 1
\end{array}\right) .
$$

Thus there are exactly two distinct ways to factor an arbitrary unimodular matrix into a three-matrix product, subject to the previously discussed restrictions on matrices with zeros as the $B$ or $C$ elements.

As a final comment on matrix factorization, we note that some matrices may be decomposed into forms involving only one each of $\alpha$ and $\beta$ type matrices. To avoid unnecessary cost in system design and fabrication, it is important to be able to recognize such simplifications when they exist. For this purpose a convenient and easily demonstrated result is the following:

THEOREM: A matrix can be decomposed using at most one $\alpha$ and one $\beta$ factor if and only if $A=1$ (the 


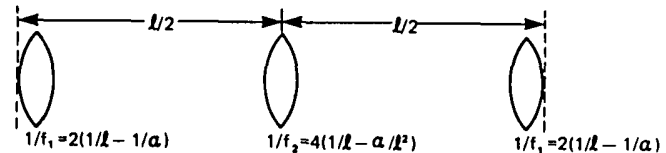

Fig. 2. Possible representation of a complex $\alpha$ type element.

order would be $\beta, \alpha$ ) or $D=A D-B C$ (the order would be $\alpha, \beta)$.

Because of the consequent simplifications, one might sometimes employ this result as a constraint in establishing the original matrix to be synthesized.

\section{Practical Realizations}

Our discussions thus far have emphasized the factorization of arbitrary $2 \times 2$ matrices into certain primitive matrix factors. It remains now to be shown that these factors can actually be represented by practical optical elements. As a starting point, one finds that any $\beta$ type matrix can be realized in practice. In particular, the product (in either order) of the matrix for a thin lens or spherical mirror with the matrix for a Gaussian transmission filter yields a $\beta$ matrix having an arbitrary complex $\beta$ element:

$$
\left(\begin{array}{cc}
1 & 0 \\
-1 / f & 1
\end{array}\right)\left(\begin{array}{cc}
1 & 0 \\
-i \lambda / \pi w_{a}^{2} & 1
\end{array}\right)=\left(\begin{array}{cc}
1 & 0 \\
-1 / f-i \lambda / \pi w_{a}^{2} & 1
\end{array}\right)=\left(\begin{array}{cc}
1 & 0 \\
\beta_{r}+i \beta_{i} & 1
\end{array}\right)
$$

It is, of course, true that the inverse Gaussian transmission characteristic $\left(w_{a}^{2}<0\right)$ cannot be maintained to arbitrary radii, but it is only necessary that this profile be approximated to the largest radius of the beam. This same restriction applies to the radial phase shift characteristics of finite diameter lenses.

The realization of arbitrary $\alpha$ matrices is a bit more complicated. The only practical matrix that is automatically of the $\alpha$ type is the matrix for a uniform medium of length $l$. But $l$ is always a positive real number, so this matrix is totally inadequate for representing the negative or complex $\alpha$ elements that might result from the factorization of an arbitrary complex matrix. For this purpose a more general representation is needed, and one possibility consists of three complex lenses separated by two uniform media. A symmetric version of such a system is shown in Fig. 2. In the figure the elements represented as thin lenses are to be understood as general $\beta$ type optical elements having complex focal lengths. As indicated previously, such elements can be easily realized from an ordinary thin lens followed (or preceded) by a Gaussian transmission filter.

For the system of interest the matrix product corresponding to Fig. 2 reduces to an $\alpha$ matrix according to

$$
\begin{aligned}
\left(\begin{array}{ll}
1 & \alpha \\
0 & 1
\end{array}\right)= & \left(\begin{array}{cc}
1 & 0 \\
2\left(\alpha^{-1}-l^{-1}\right) & 1
\end{array}\right)\left(\begin{array}{cc}
1 & l / 2 \\
0 & 1
\end{array}\right)\left(\begin{array}{cc}
1 & 0 \\
4\left(\alpha l^{-2}-l^{-1}\right) & 1
\end{array}\right) \\
& \times\left(\begin{array}{cc}
1 & l / 2 \\
0 & 1
\end{array}\right)\left(\begin{array}{cc}
1 & 0 \\
2\left(\alpha^{-1}-l^{-1}\right) & 1
\end{array}\right) .
\end{aligned}
$$

Thus an arbitrary complex $\alpha$ matrix can be represented as a product of five realizable factors, and this result can be easily checked by multiplication.

To illustrate the use of Eq. (40), let us imagine that we are trying to find a practical realization for a given unimodular $A B C D$ matrix using the factorization given in Eq. (38). With good luck each of the resulting three matrix factors can be realized by a single optical element (or perhaps two elements, depending on how one fabricates a combination of a lens and Gaussian aperture). Thus only three elements are required. With bad luck, however, the $B$ element of the $\alpha$ matrix may not be a positive real number. Then a more complicated representation of the $\alpha$ matrix is required, and the fiveelement system shown in Fig. 2 works well. The initial and final lenses of this system may be combined with the initial and final lenses implies by the $\beta$ matrices in Eq. (38), so the total number of elements used in such a decomposition is five. Since the factorization in Eq. (37) involves two $\alpha$ matrices, with bad luck this alternative procedure could lead to six or nine optical elements.

The previous remarks have implied that it is unfortunate when one encounters an $\alpha$ matrix in which the $B$ element is not real and positive. However, a highly desirable feature of the expansion shown in Fig. 2 is that the distance $l$ between the reference planes is totally arbitrary. In a practical situation one might like to specify the length of the optical system which is to produce a desired beam transformation. In the simpler three-matrix realizations where an expansion like that shown in Fig. 2 is not required, there is no length flexibility.

The emphasis so far in this section has been on the possibility of obtaining practical realizations for arbitrary $\alpha$ and $\beta$ matrices. Relatively little needs to be said about realizing $\gamma$ matrices. As mentioned previously, these matrices only occur if the output reference plane involves a medium with different refracting properties from the input reference plane. In such cases it seems probable that the matrix determinant and hence the $\gamma$ matrix will have been specified at the outset. Otherwise one might discover at the end of the synthesis process that the desired output beam occurs within an undesirable refracting medium.

\section{Example: The Identity System}

The concepts developed in the previous sections can be illustrated by considering as an example the identity optical system. The identity matrix is a diagonal matrix in which the diagonal elements are equal to unity. It follows from Eq. (1) that the effect of the identity matrix in ray optics is that it leaves the position and slope of a light ray unchanged. In beam optics, one finds from Eq. (4) that the identity matrix produces no change in the beam spot size or phase front curvature. A reasonable question to ask is whether a nontrivial identity optical system can be fabricated from realizable optical components. In fact, one finds that there are infinitely many such systems. 


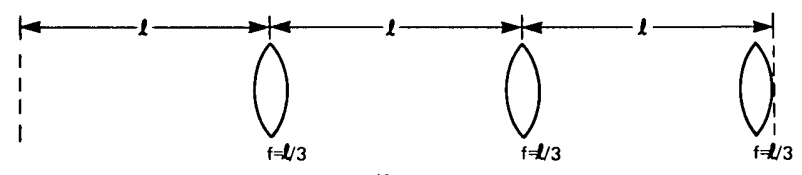

(A)

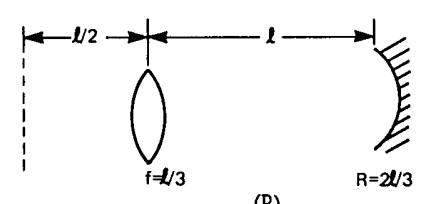

(B)

Fig. 3. Identity systems for (A) transmission and (B) reflection of rays or beams.

The identity matrix is a special case of the diagonal matrices considered previously. Thus Eq. (33) would suggest the factorization

$$
\left(\begin{array}{ll}
1 & 0 \\
0 & 1
\end{array}\right)=\left(\begin{array}{cc}
1 & -l_{1} \\
0 & 1
\end{array}\right)\left(\begin{array}{cc}
1 & l_{1} \\
0 & 1
\end{array}\right)
$$

where the second matrix represents free space of length $l_{1}$. The first matrix can be decomposed using Eq. (40), and the final result is

$$
\begin{aligned}
\left(\begin{array}{ll}
1 & 0 \\
0 & 1
\end{array}\right)= & \left(\begin{array}{cc}
1 & 0 \\
-2\left(l_{1}^{-1}+l_{2}^{-1}\right) & 1
\end{array}\right)\left(\begin{array}{cc}
1 & l_{2} / 2 \\
0 & 1
\end{array}\right)\left(\begin{array}{cc}
1 & 0 \\
-4\left(l_{1} l_{2}^{-2}+l_{2}^{-1}\right) & 1
\end{array}\right)\left(\begin{array}{cc}
1 & l_{2} / 2 \\
0 & 1
\end{array}\right) \\
& \times\left(\begin{array}{cc}
1 & 0 \\
-2\left(l_{1}^{-1}+l_{2}^{-1}\right) & 1
\end{array}\right)\left(\begin{array}{cc}
1 & l_{1} \\
0 & 1
\end{array}\right) .
\end{aligned}
$$

The length $l_{2}$ in this expansion is, as noted previously, completely arbitrary, but the results look most elegant if we choose the relationship $l_{2}=2 l_{1}=2 l$. Then Eq. (42) simplifies to

$$
\left(\begin{array}{ll}
1 & 0 \\
0 & 1
\end{array}\right)=\left(\begin{array}{cc}
1 & 0 \\
-3 / l & 1
\end{array}\right)\left(\begin{array}{ll}
1 & l \\
0 & 1
\end{array}\right)\left(\begin{array}{cc}
1 & 0 \\
-3 / l & 1
\end{array}\right)\left(\begin{array}{ll}
1 & l \\
0 & 1
\end{array}\right)\left(\begin{array}{cc}
1 & 0 \\
-3 / l & 1
\end{array}\right)\left(\begin{array}{ll}
1 & l \\
0 & 1
\end{array}\right)
$$

The experimental setup corresponding to this result is sketched in Fig. 3(A). Except for translation along the optical axis, a light ray or Gaussian beam leaving this system will be identical to the light ray or Gaussian beam as it entered the system.

The identity system just described operates on transmitted rays and beams. It is also possible to synthesize a reflective identity system, and an easily verified example is given in Fig. 3(B). This system would behave in a manner identical to a flat mirror located at the reference plane, and we have confirmed this behavior in visual and $\mathrm{He}-\mathrm{Ne}$ laser experiments. As a practical application, such a system could be used as the end mirror on a waveguide laser. ${ }^{9}$ A flat waveguide mirror is the ideal, but practical problems often force one to position the mirror away from the end of the waveguide. The field distribution emerging from the waveguide can be expanded in Hermite-Gaussian modes, and it follows from Eqs. (4), (9), and (10) that for an identity reflector the amplitude and phase distributions of the reentering fields would be exactly the same as if the waveguide had been terminated by a flat mirror.

\section{Conclusion}

The techniques for analyzing the propagation of light rays and Gaussian beams through paraxial optical systems are well known. The purpose of this study has been to develop systematic methods for the opposite process of synthesis, the design of an optical system which will produce some desired transformation of a ray or beam. The synthesis process, as developed here, involves three more-or-less distinct steps: (1) converting the desired performance characteristics of an optical system into explicit values or constraints on the values of the transformation matrix elements; (2) factoring the matrix into certain primitive matrix forms; and (3) replacing each of these primitive matrices with realizable optical components. In most cases this process is straightforward and systematic, and it is also well suited for computer calculation.

The author is pleased to acknowledge valuable discussions with H. J. Orchard and J. Vetrovec.

\section{References}

1. For an early review of this subject see H. W. Kogelnik and T. Li, Appl. Opt. 5, 1550 (1966)

2. H. W. Kogelnik, Bell Syst. Tech. J. 44, 455 (1965).

3. H. W. Kogelnik, Appl. Opt. 4, 1562 (1965).

4. L. W. Casperson and A. Yariv, Appl. Phys. Lett. 12, 355 (1968).

5. L. W. Casperson and S. D. Lunnam, Appl. Opt. 14, 1193 (1975) and references therein.

6. L. W. Casperson, J. Opt. Soc. Am. 66, 1373 (1976).

7. L. W. Casperson, IEEE J. Quantum Electron. QE-10, 629 (1974).

8. A. E. Siegman, IEEE J. Quantum Electron. QE-12, 35 (1976).

9. J. J. Degnan, Appl. Phys. 11, 1 (1976) and references therein. 